\title{
Implementation of the ISO/IEC 24756 for the Interaction Modeling of an AAL Space
}

\author{
Pilar Sala, Carlos Fernandez, Juan Bautista Mocholí, \\ Pablo Presencia, and Juan Carlos Naranjo \\ Instituto de Aplicaciones de las Tecnologías de la Información y de las Comunicaciones \\ Avanzadas. ITACA Technological Centre, \\ Universidad Politécnica de Valencia, Edificio 8G \\ Camino de Vera s/n 46022 Valencia, Spain \\ \{msalaso, carferll, juamocag, pabpreor, jcnaranjo\} aitaca.upv.es
}

\begin{abstract}
This paper presents the results of the implementation of an accessibility verifier tool for AAL systems using the ISO/IEC 24756 standard. The accessibility verifier tool is based in an interaction model where the Common Accessibility Profile (CAP), as defined by the standard, is used to perform the checking of the accessibility constrains of the system configuration against the user capabilities. The paper gives information of the major design decisions in developing the tool, the context of use in the VAALID project and the relation with the standard.
\end{abstract}

Keywords: AAL Systems, Accessibility verifier, Common accessibility Profile, ISO/IEC 24756, interaction modeling.

\section{Introduction}

AAL is a concept which aims to prolong the time people can live decently in their own homes by increasing their autonomy and self-confidence, the discharge of monotonous everyday activities, or monitoring and caring for the elderly or ill person, in order to enhance their security and save resources [1]. AAL embraces all the technological challenges in the context of Ambient Intelligence to face such problem. A common term used when referring to AAL technologies is the "AAL space" or the physical space, the environment, in where the AAL products and services are deployed. The AAL space is a core component of any AAL solution and may be seen as the sum of sensors, actuators and user interface devices through which the user interacts with.

The user interaction with the AAL services must be carefully designed by the usability engineers. AAL spaces refers to electronic environments that are sensitive and responsive to the presence of people and provide assistive propositions for maintaining an independent lifestyle, so here the challenge for the designer is to experiment with new and innovative modalities of interaction that must be, of course, accessible 
and usable[2]. Some examples of the different interaction modalities between the user and the AAL environment are speech recognition systems, gestural systems, contextsensitive systems, visual interaction, auditive interaction, tactile interaction, etc.

When designing such AAL spaces there is a need to model the context in which the user is involved, being this need one of their main goals. The information managed by context is related to the environment, to the user and to the devices plus a description of available services. Among others objectives for the modeling, the evaluation of the interaction between the environment and the user in terms of the accessibility facets and, more concretely, the information related to the interaction capabilities and its accessibility is a very important need to cover.

This paper describes the implementation of the ISO/IEC 24756[3] for the interaction modelling of an AAL space. The recently approved standard has been used to define a Common Accessibility Profile (CAP) on the topic of defining interaction and accessibility, and in order to deal with the description of all the possible features of a user and the functionalities provided by devices and systems in terms of accessibility constraints. The CAP concept as presented by Fourney[4] and later adopted as a basis for the standard has been used by the VAALID project to perform the accessibility constrains verification.

In the next section an introduction to VAALID project can be found. The main objective of the VAALID project is to develop a 3D-Immersive Simulation Platform for computer aided design and validation of User-Interaction subsystems that improve and optimize the accessibility features of Ambient Assisted Living services for the social inclusion and independent living.

The paper describes in details the results of the implementation and how is being used in the framework of the VAALID project.

\section{The Accessibility Constrains Verification in the VAALID Project}

VAALID project [5] aims at creating new tools and methods that facilitate and streamline the process of creation, design, construction and deployment of accessible technological solutions for Ambient Intelligence to face the problem of the aging population.

These tools support the adoption of Human Centered Design methodology to create AAL solutions that are easily accessible for the user, technically and economically viable for the producers, and affordable and sustainable for the welfare system.

To fulfill this vision, VAALID project addresses the development of an Integrated Development Environment (IDE) for the designers of AAL solutions. VAALID IDE focuses on the design of the interaction between an elderly (called "beneficiary" in VAALID project context) and the AAL solution. Also, VAALID IDE provides the designer with tools to evaluate the accessibility and usability aspects of such interactions. 
VAALID IDE consists of two environments that provide tools to the designer:

- An Authoring Environment (AE), that allows the designers to create and deploy the components that constitute the interaction structure of an AAL solution.

- An immersive Simulation Environment (SE), that allows the designers to simulate previously created AAL solutions; and the beneficiaries to experience the AAL solutions in virtual and augmented reality.

VAALID IDE target users are the professionals who are in charge of the conception, design, testing and validation of the human aspects of AAL solutions. They are collectively defined as AAL solution designers in VAALID project context.

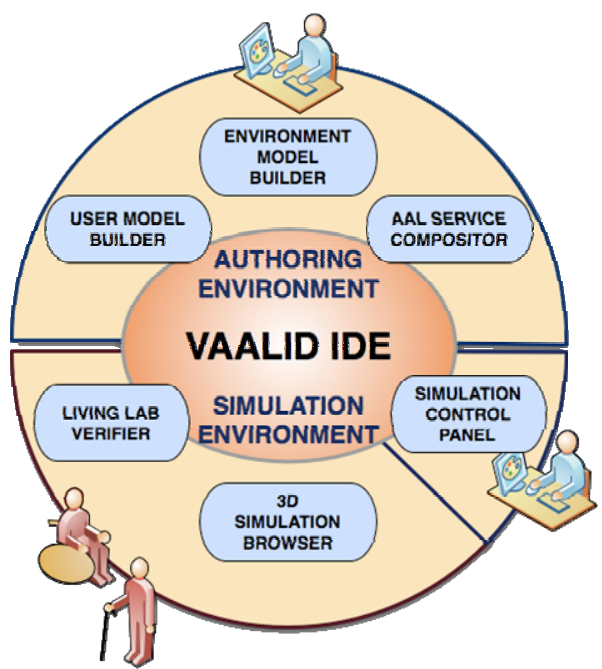

Fig. 1. VAALID IDE Tools

The VAALID IDE supports the AAL designer in adopting a Human Centered Design (HCD) process for the development of AAL Services. It is a kind of a rapiddesign-prototyping tool that allows an iterative development of AAL solutions embedded into the HCD process. It empowers designers to create AAL solutions of a higher quality faster, easier and inexpensively by giving attention to the needs and limitations of the end users at each stage of the design process.

The accessibility constraints verification done with the Accessibility verifier is used during the design and implementation phase of an AAL HCD process and it allows the designer to automatically detect accessibility issues derived from matching accessibility profiles of the virtual beneficiaries with the different elements included in the simulation. 


\section{ISO/IEC 24756. Common Accessibility Profiles and VAALID Interaction Model}

The Accessibility Verifier is built following the guidelines described in the ISO/IEC 24756 standard. This International Standard introduces a model of accessibility as a basis for understanding access issues with the interactions between users and systems in various environments. The model shows that users and systems must share capabilities of communicating. The standard provides a framework to specify a profile of common access capabilities (the CAP) of interactive systems, users, and their environment that are necessary for accessibility to be possible. [3]

The CAP is a key concept in the VAALID project since it is the base for the interaction model. This model was developed to collect the concepts that describe the capabilities of each element present in the interaction being developed by the usability engineer. The interaction model is the bridge or link between the user model (the model that describes the user abilities, preferences, etc) and the environment model (the model that collects the concepts related to elements presents, devices, dimensions, environmental conditions (lighting, humidity, noise, temperature, etc.)).

The CAP and the interaction model are the mechanisms used to match the user's interaction capabilities against the interaction capabilities of the devices, sensors and user interfaces. The standard defines the Interacting Components (ICs) as the actors of any interaction communication. In VAALID the ICs are the users, the devices and the environmental conditions that are part of an AAL Solution so the Overall CAP is the sum of the $\mathrm{CAP}_{\mathrm{USE}}+\mathrm{CAP}_{\mathrm{SYS}}+\mathrm{CAP}_{\mathrm{ENV}}$ (VAALID is not considering Assistive Technologies). The following figure shows an example of the matching between the CAPS with the nomenclature used in VAALID.

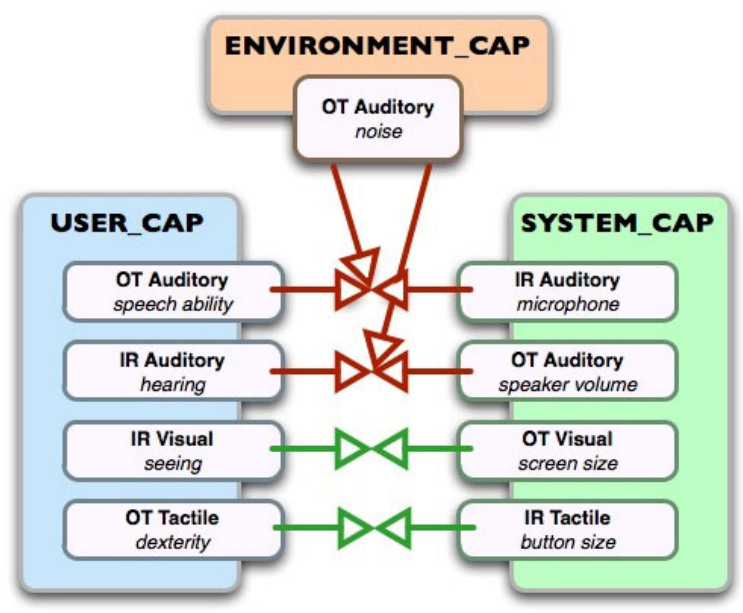

Fig. 2. Example of matching CAPs 
The CAPs have been implemented as ontology classes. The ontology classes represent the hierarchy and relationships between the CAP, the ICs, the Component Features (CFs) and the Type specific information (i.e modality $\left(\mathrm{CAP}_{\mathrm{M}}\right)$, capability $\left(\mathrm{CAP}_{\mathrm{C}}\right)$, and processing $\left.\left(\mathrm{CAP}_{\mathrm{P}}\right)\right)$. See the standard for a detailed explanation of these terms.

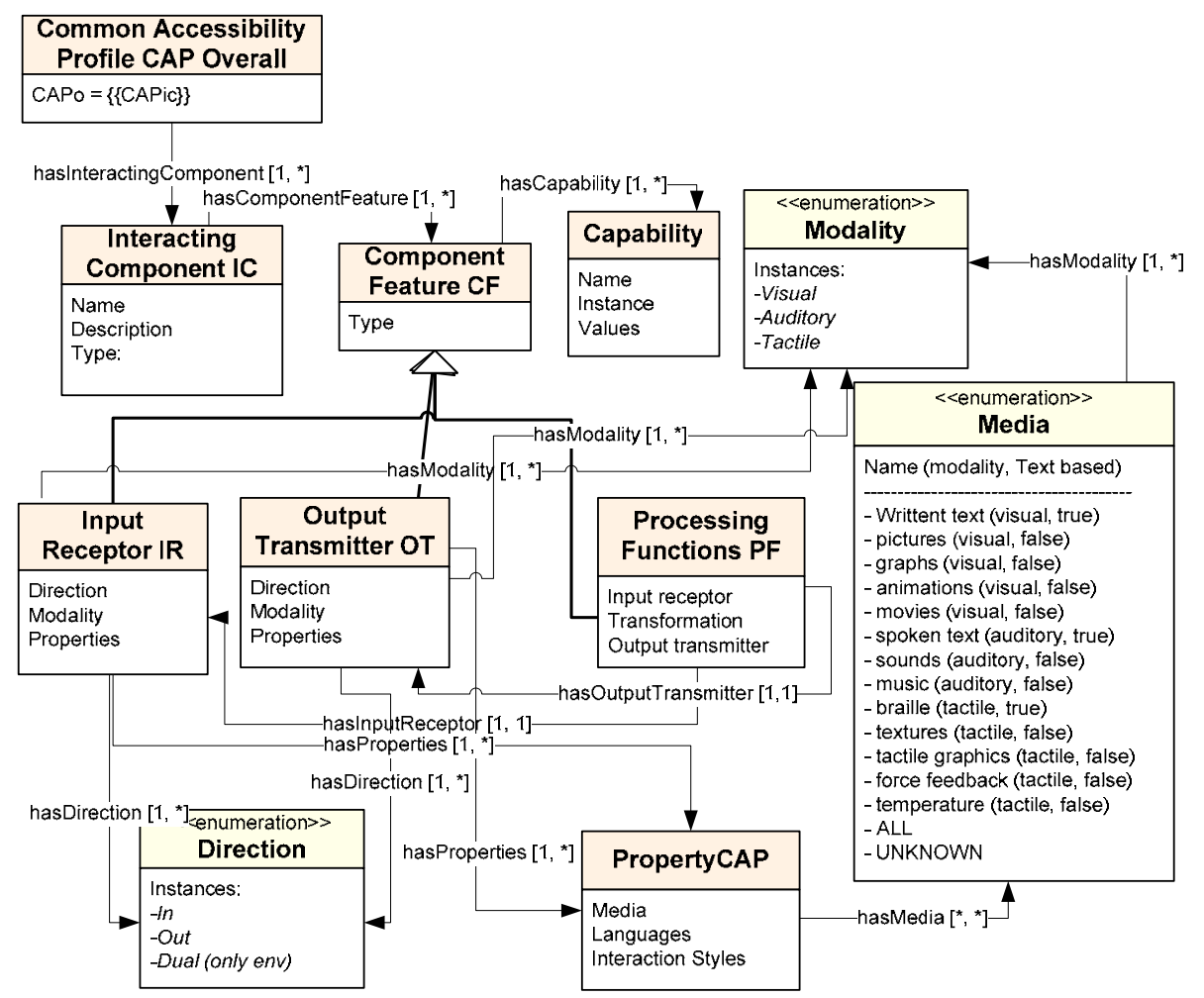

Fig. 3. Interaction Model

In the following tables the different entities and the relationships of the ontology are described following the structure described in the standard.

Table 1. CAP

\section{Relations}

Has Interacting Component $\rightarrow$ Interacting Component: A CAP overall has at least one Interacting component. 
Table 2. ICs

\begin{tabular}{l}
\hline Attributes \\
\hline Name: A string defining the context of use of the service. \\
\hline Description: A string with the description of the service. \\
\hline Relations \\
\hline Has Component Feature $\rightarrow$ Component Feature: An interacting component has at least one \\
Component Feature. \\
\hline Has CAP type $\rightarrow$ CAPType: Defines the type of the IC described: CAPuse (user), CAPsys \\
(system), CAPat (assistive technology), CAPenv (environment)
\end{tabular}

Table 3. CFs

\section{Relations CF}

Has Capability $\rightarrow$ Capability: Optional information that it's used to set a certain capability of the element described.

\section{Relations Input Receptor (IR), Output Transmitter (OT)}

Has Interaction component $\rightarrow$ Interaction Component: Optional information. The CAP of an IR could be composed by other ICs.

Has Direction $\rightarrow$ Direction. To set the direction.

Has Modality $\rightarrow$ Modality Sets the modality of the IR.

Has Property $\rightarrow$ PropertyCAP. defines the properties (not for capabilities) of a IR/OT.

\section{Results: The Accessibility Verifier}

The main goal of Vaalid Accessibility Verifier is to check that the interaction capabilities of the environment, spaces and devices are adequate to the interaction requirements of the user. In order to perform this checking, the Vaalid Accessibility Verifier is based on the interaction model described above.

The user model is the basis for creating the CAP $\mathrm{USE}_{\text {. The }} \mathrm{CAP}_{\mathrm{USE}}$ (or USER_CAP class) encapsulates the accessibility capabilities of the user model representing the user target for the validation.

In the same way, the interaction capabilities of the environment and spaces are also defined. Each device is modeled and specified in the environment model ${ }^{1}$ and consti-

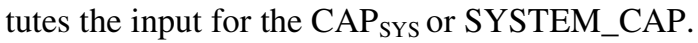

\subsection{Entry Description}

The information that is needed for the verification is defined and stored in the ontology classes (Fig. 3). Fig. 4 shows the high level class diagram used to implement the

${ }^{1}$ The environment model in VAALID must not be confused with the $\mathrm{CAP}_{\mathrm{ENV}}$. The environment model represents the space, sensors or user interface devices that are part of the simulation. $\mathrm{CAP}_{\mathrm{ENV}}$ defines the conditions that can reduce the accessibility of a system. 
CAP and the related concepts (for simplicity only visual capabilities are shown). Each CAP defined for each interacting component (IC) includes a set of component features (CF). In addition, the CFs have a collection of capabilities (CP) which increases the accuracy of the given information for every feature.

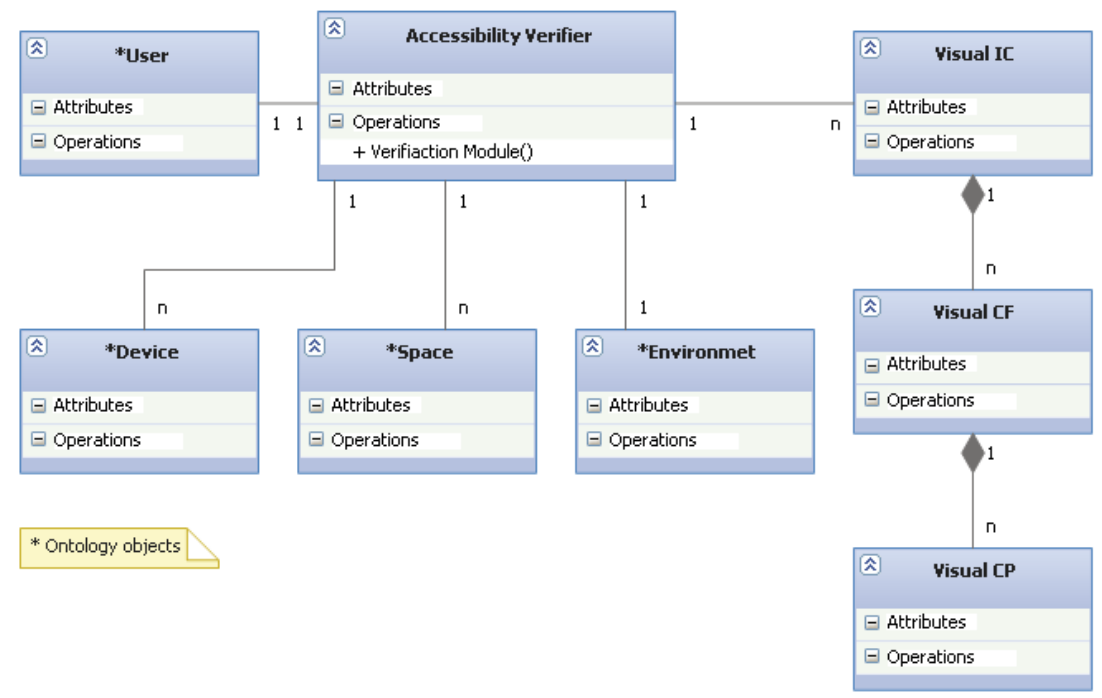

Fig. 4. Accessibility Verifier main classes diagram

A CF include the information regarding the direction ("In" or "Out"), defines a language, a modality ("Tactile", "Visual" or "Auditory"), and finally specifies if a media is used and which one.

CPs provide information about the units, maximums and minimums values used in the $\mathrm{CF}$.

In the current implementation of the Accessibility Verifier, some features are created automatically on specific CFs. The remaining features, due to the amount and complexity of the elements, must be entered manually by creating the proper CFs. For instance, we can model a user with a hearing impairment who is unable to hear bellow $60 \mathrm{~dB}$ (Fig. 5) and also model a space that contains a loudspeaker configured to produce sound between 60 and $120 \mathrm{~dB}$.

\subsection{Data Processing}

Once the entries are introduced, information regarding the ICs of all the elements is stored in the ontology classes. The consistency of the information is then checked since it is possible to rule out any inner discrepancy.

The main feature of the Accessibility Verifier module is to check the accessibility for all the CFs that have been defined in the project based on specific rules. User's CFs are compared one by one against the CFs of the remaining items (environment, spaces and devices), paying particular attention to the criteria based on direction and modality of such items. 


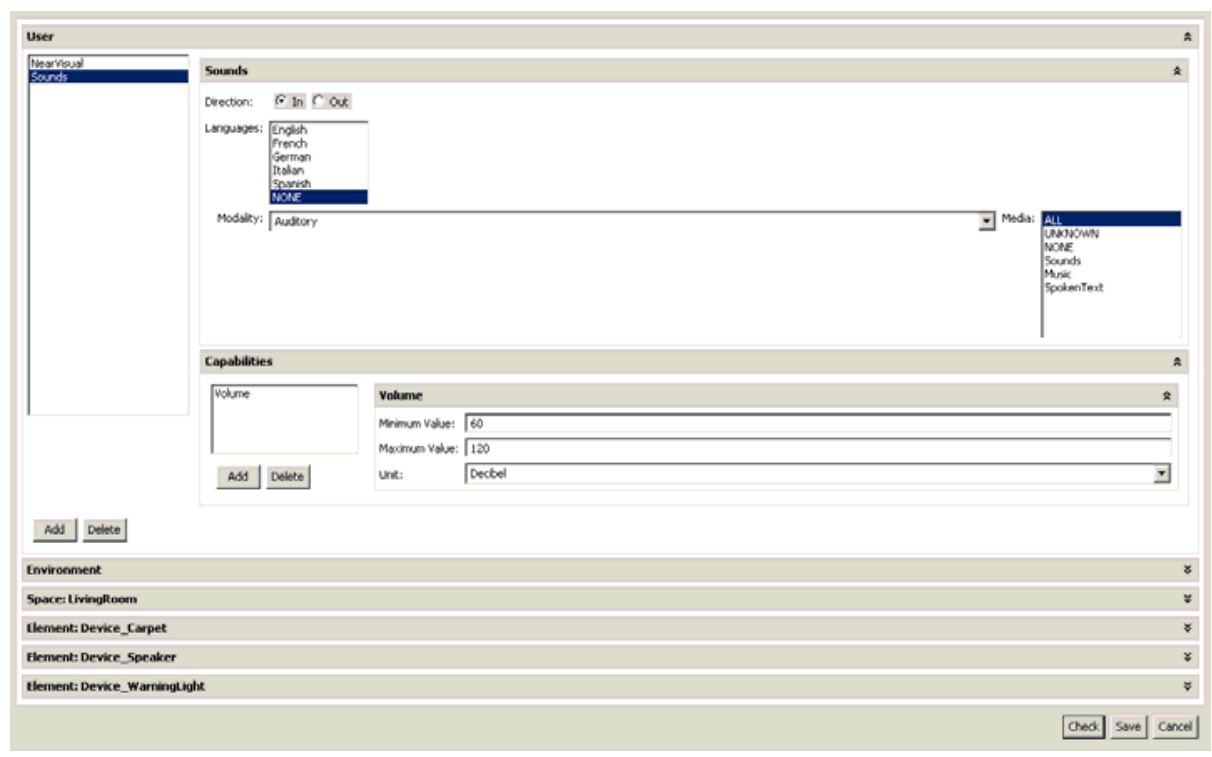

Fig. 5. User's CF

If during the checking no items are found satisfying the condition of the CF, the unmatched feature is registered to be prompt. In this step the module verifies that every item in the environment fulfills the needs of the user. In fact, the AV verifies that the user is able to interact with the device. In the previous example we are ensuring that the user will hear the fire alarm in case it is triggered.

\subsection{Outputs}

The Accessibility Verifier shows a message according the evaluation done in the verification module. Three options are possible.

The first one indicates that "there is nothing to evaluate". This is possible because there is no definition of a user and/or he has no $\mathrm{CF}$, or it could be because the present elements in the application have no defined $\mathrm{CF}$, and therefore there is no possible evaluation for the system.

The second message indicates that "no errors have been detected". That means that for each $\mathrm{CF}$ of the user, there is at least a $\mathrm{CF}$ in one of the elements (environment, spaces and devices) with the same modality and opposite direction. Backwards is the same, for each CF of the elements on the Project the user has to have at least one CF with the same modality but opposite direction.

The third message shows us that "some accessibility issues have been detected". This is the more important result since it gives us a clear indication that an accessibility problem is arising in the proposed AAL solution. It also provides information about the specific accessibility issues with the current configuration of the AAL system. It retrieves the registered unmatched features to help to the resolution of those accessibility issues (Fig. 6). 


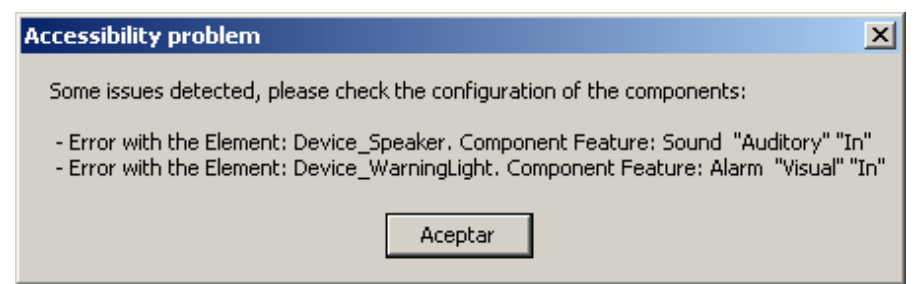

Fig. 6. Verification output

\section{Conclusions}

The Accessibility verifier is an important tool in hands of the designer to ensure that there are no major accessibility issues in the solution he or she is testing. The use of the standard provides a solid framework for the development of the module and guarantees the maintenance and extension of future versions of the tool. The standard can also be used in order to formalize the exchange of CAPs between different platforms or simulation tools.

Although in this first version the verification can be considered just as an indication of potential accessibility problems, it has been proven as a useful tool in the assessment of complex systems when a lot of devices are deployed in the AAL space. It allows the designer to rely on this verification for avoiding accessibility problems and concentrate in the solution itself. The designer does not longer need to care about the increasing number of interaction parameters and the different user interaction profiles that are used during the verification of this type of systems.

This tool, in conjunction with the rest of the VAALID IDE, allows the designer to create an AAL solution with no prior knowledge about the different facets of a disability; no special expertise is required for designing the system since the tool supervise the accessibility constrains that must be taken into account.

The design of AAL spaces free of accessibility constrains contributes to the AAL paradigm of solutions that fit the persons' needs and improve their dignity and quality of life.

In the time of writing this paper (January 2011) only preliminary results are available; the validation phase will conclude in April 2011 and some results will be given in the presentation of the paper in July 2011. The preliminary results give us a great support to continue this research.

Future work must be focused in the development of more intelligent algorithms to provide a more sophisticated and powerful verification. The automatic extraction of data from the user and system models is also a field for further research.

Acknowledgments. This work has been partially funded by the European Union in the context of the VAALID project. Our gratitude to the VAALID consortium composed of the following partners: Fh-IGD, UniPR, VOLTA, UPM, UID, SPIRIT and ITACA. 


\section{References}

1. Steg, H., Strese, H., Hull, J., Schmidt, S.: Europe is facing a demographic challenge Ambient Assisted Living offers solutions (2005)

2. Naranjo, J.-C., Fernandez, C., Sala, P., Hellenschmidt, M., Mercalli, F.: A modelling framework for ambient assisted living validation. In: Stephanidis, C. (ed.) UAHCI 2009. LNCS, vol. 5615, pp. 228-237. Springer, Heidelberg (2009)

3. ISO/IEC 24756. Information technology - Framework for specifying a common access profile (CAP) of needs and capabilities of users, systems, and their environments. First edition 2009-04-01

4. Fourney, D.: Using a common accessibility profile to improve accessibility. Master Thesis submitted to the College of Graduate Studies and Research, University of Saskatchewan, Saskatoon, Canada (2007)

5. http: //www. vaalid-project.org/ 